\title{
Pound-Drever-Hall error signals for the length control of three-port grating coupled cavities
}

\author{
Michael Britzger, ${ }^{1}$ Daniel Friedrich, ${ }^{1}$ Stefanie Kroker, ${ }^{2}$ Frank Brückner, ${ }^{2}$ \\ Oliver Burmeister, ${ }^{1}$ Ernst-Bernhard Kley, ${ }^{2}$ Andreas Tünnermann, ${ }^{2}$ \\ Karsten Danzmann, ${ }^{1}$ and Roman Schnabel ${ }^{1, *}$ \\ ${ }^{1}$ Albert-Einstein-Institut, Max-Planck-Institut für Gravitationsphysik and Leibniz Universität Hannover, \\ Callinstrasse 38, 30167 Hannover, Germany \\ ${ }^{2}$ Institut für Angewandte Physik, Friedrich-Schiller-Universität Jena, Max-Wien-Platz 1, 07743 Jena, Germany \\ ${ }^{*}$ Corresponding author: roman.schnabel@aei.mpg.de
}

Received 10 November 2010; revised 18 February 2011; accepted 27 February 2011; posted 17 March 2011 (Doc. ID 137978); published 22 July 2011

\begin{abstract}
Gratings enable light coupling into an optical cavity without transmission through any substrate. This concept reduces light absorption and substrate heating and was suggested for light coupling into the arm cavities of future gravitational wave detectors. One particularly interesting approach is based on allreflective gratings with low diffraction efficiencies and three diffraction orders (three ports). However, it was discovered that, generally, three-port grating coupled cavities show an asymmetric resonance profile that results in asymmetric and low quality Pound-Drever-Hall error signals for cavity length control. We experimentally demonstrate that this problem is solved by the detection of light at both reflection ports of the cavity and the postprocessing of the two demodulated electronic signals. (C) 2011 Optical Society of America
\end{abstract}

OCIS codes: $\quad 050.1950,050.2230,120.2230,120.3180,230.1950$.

\section{Introduction}

In recent years an international network of laserinterferometric gravitational wave detectors has been developed [1-4]. These detectors are operating at sensitivities that allow a direct detection of gravitational waves caused by astrophysical events. The network of detectors is currently in a state of enhancement and development toward the second generation. Simultaneously, new interferometer concepts are evaluated for future detectors such as the European Einstein Telescope [5].

To improve the shot-noise-limited sensitivity, future detectors will operate with ultrahigh-power lasers and coupled optical resonators, resulting in stored light powers of up to the megawatt range [5]. Residual optical absorption within the bulk materials of the transmissive components causes thermal

0003-6935/11/224340-07\$15.00/0

(C) 2011 Optical Society of America lensing and photon absorption induced thermorefractive noise [6,7]. Temperature-dependent $Q$ factors of the current transparent materials and the remaining absorption make their cryogenic cooling challenging $[8,9]$.

A promising technique proposed to improve the sensitivity of future detectors is the replacement of the transmissive optical components by alternatives based on dielectric reflection gratings $[10,11]$. The resulting all-reflective interferometers are beneficial because they reduce the impact of all thermal issues that are associated with absorbed laser power in optical substrates, and they allow the use of opaque materials with favorable mechanical and thermal properties.

The all-reflective component that meets the demands as a potential diffractive coupling component to the arm cavity of a Michelson-type interferometer is the so-called three-port grating with three orders of diffraction [12,13]. Using one diffraction order as the coupling port to the cavity, the two remaining 
diffraction ports show a characteristic output power behavior with respect to the balance of the diffraction efficiencies and the tuning of the cavity. In particular for three-port gratings with diffraction efficiencies different from their allowed extremal values, the shapes of the resonance peaks of the two remaining ports are not symmetric with respect to the tuning of the cavity. This affects the Pound-Drever-Hall (PDH) error signals [14] that are needed for stabilization of the cavity and may deteriorate the cavity control quality. Since a three-port grating cavity provides two correlated ports where the phase-modulated sidebands are reflected, the electronically added signals at these two ports sum up to a symmetrical PDH signal that is suitable for stable cavity control.

In order to verify that effect and also to validate the three-port grating description for strong asymmetries and the resulting asymmetric error signals, a customized dielectric three-port grating was manufactured. Experimentally (see Fig. 1) it is shown that an appropriate error signal can be generated by extracting signals at both reflection ports of a three-port grating coupled cavity with electronic addition of the demodulated signals. The symmetrical PDH signal provides the stable length control of resonators with three-port gratings of any balance of the diffraction efficiencies of the zeroth and second diffraction order. The experimentally generated error signals are compared to calculated signals.

\section{Three-Port Grating Coupled Cavities}

The three-port grating has three orders of diffraction $(m=0,1,2)$ with the diffraction efficencies $\eta_{0}, \eta_{1}$ and $\eta_{2}$, respectively. Using monochromatic laser light, it can be mounted in second-order Littrow configuration, where the angle of the second diffraction order is equal to the angle of the incident light $[12,13]$. As shown in Fig. 2, the first order of diffraction provides the coupling to a cavity perpendicular to the grating surface. Hence, the three-port grating relies on low

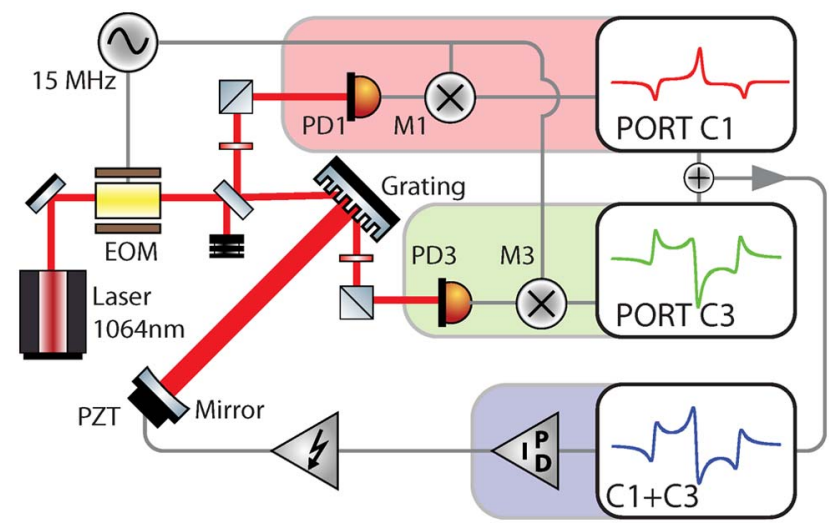

Fig. 1. (Color online) Experimental setup. The grating coupled cavity was set up in second-order Littrow configuration. At the two reflection ports (C1, C3) signals were detected with photo diodes (PD1, PD3) and demodulated (M1, M3). The sum-signal provided a proper PDH error signal that was amplified and fed back via an electronic filter to the PZT of the cavity end mirror for cavity length locking.
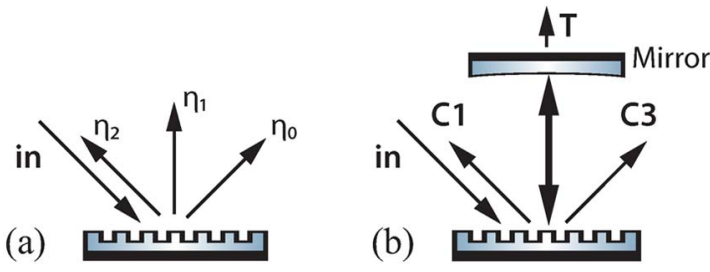

Fig. 2. (Color online) (a) Three-port grating in second-order Littrow configuration. According to each diffraction order, the amplitude efficiencies are labeled $\eta_{0}, \eta_{1}$, and $\eta_{2}$. (b) Three-port grating as coupler to an optical cavity. The two reflection ports are labeled C1 and $\mathrm{C} 3$, and the transmission port at the end mirror, $\mathrm{T}$.

first-order diffraction efficiency, which can be achieved by shallow grating structures and standard coating techniques to provide high reflectivity. This approach is therefore suited for low-loss couplers to high-finesse cavities, a stringent requirement in high-power laser interferometry. Since the grating provides three orders of diffraction, each input field couples to three output fields, and therefore the binary structured diffractive three-port component is represented by a $3 \times 3$ scattering matrix

$$
\mathbf{S}_{3 p}=\left(\begin{array}{lll}
\eta_{2} \exp \left(i \phi_{2}\right) & \eta_{1} \exp \left(i \phi_{1}\right) & \eta_{0} \exp \left(i \phi_{0}\right) \\
\eta_{1} \exp \left(i \phi_{1}\right) & \rho_{0} \exp \left(i \phi_{0}\right) & \eta_{1} \exp \left(i \phi_{1}\right) \\
\eta_{0} \exp \left(i \phi_{0}\right) & \eta_{1} \exp \left(i \phi_{1}\right) & \eta_{2} \exp \left(i \phi_{2}\right)
\end{array}\right)
$$

where $\eta_{m}$ and $\phi_{m}$ are the amplitude diffraction efficiencies and the phase changes on the diffraction for $m=0,1,2$, and $\rho_{0}$ is the amplitude reflectivity at normal incidence.

From the requirement that $\mathbf{S}_{3 p}$ needs to be unitary, the phases can be calculated, yielding the following set of phases:

$$
\begin{aligned}
& \phi_{0}=0 \\
& \phi_{1}=-(1 / 2) \arccos \left[\left(\eta_{1}^{2}-2 \eta_{0}^{2}\right) /\left(2 \rho_{0} \eta_{0}\right)\right], \\
& \phi_{2}=\arccos \left[-\eta_{1}^{2} /\left(2 \eta_{2} \eta_{0}\right)\right]
\end{aligned}
$$

Since the phases are real numbers, the absolute value of the argument of the arccos must be smaller than or equal to one. Therefore, there are an upper and lower limit for $\eta_{0}$ and $\eta_{2}$, which are given by

$$
\eta_{0_{\min }^{\max }}=\eta_{2_{\min }^{\max }}=\left(1 \pm \rho_{0}\right) / 2 .
$$

As a consequence, a binary three-port diffraction grating can only be designed and manufactured with diffraction efficiencies within these boundaries. In addition, the phase shifts depend on the diffraction efficiencies. For a fixed first-order diffraction efficiency, they are determined by the balance of the diffraction efficiencies of the zeroth and second diffraction order. In [13] these properties of a threeport grating coupled cavity have been investigated, and the amplitude fields at the backward reflected 
field $c_{1}$, the intracavity field $c_{2}$, the forward reflected field $c_{3}$, and the transmitted field at the mirror $t$ have been described by

$$
\begin{aligned}
& c_{1}=\eta_{2} \exp \left(i \phi_{2}\right)+\eta_{1}^{2} \rho_{1} \exp \left(i\left[2\left(\phi_{1}+\Phi\right)\right]\right) d, \\
& c_{2}=\eta_{1} \exp \left(i \phi_{1}\right) d, \\
& c_{3}=\eta_{0}+\eta_{1}^{2} \rho_{1} \exp \left(i\left[2\left(\phi_{1}+\Phi\right)\right]\right) d, \\
& t=i \tau_{1} \exp (i \Phi) c_{2},
\end{aligned}
$$

where $\Phi$ represents the phase acquired after one round trip of the cavity, and the resonance factor is defined by $d=\left(1-\rho_{1} \rho_{2} e^{2 i \Phi}\right)^{-1}$.

In [15] it has been shown that for a three-port grating as coupler to optical cavities, the balance of $\eta_{0}$ and $\eta_{2}$ affects the outcoupled power and the shape of the resonance peaks at the two reflection ports $\mathrm{C} 1$ and C3 of the cavity. Changes of the balance of $\eta_{0}$ and $\eta_{2}$ of a three-port grating always lead to a change of the grating cavity power output characteristics but do not influence the bandwidth of the intracavity field [16].

\section{Pound-Drever-Hall Error Signals}

In order to stabilize the length of a resonator, the $\mathrm{PDH}$ technique is commonly used [14]. In this scheme, phase-modulated sidebands are imprinted on the light field before it enters the resonator. In general, a sideband frequency of several megahertz outside the linewidth of the cavity is chosen. The light that is reflected at the cavity is detected by a photodetector and demodulated at the modulation frequency. The resulting error signal is a measure of the mismatching between the frequency of an eigenmode of the cavity and the frequency of the incident carrier light. Apart from being a measure of the detuning, it also contains information about the sign of the detuning. The resonance condition of the cavity can thus be maintained by feeding this signal back to an actuator via an appropriate electronic filter.

In principle the three-port grating cavity has three ports $(\mathrm{C} 1, \mathrm{C} 3, \mathrm{~T})$ where a control signal can be extracted. The incident light beam is phase modulated with the frequency $\omega_{m}$, which generates upper and lower sidebands of the carrier light $\omega_{0}$. They are described in analogy to a linear Fabry-Perot resonator. The transfer functions of the carrier are given in Eqs. (6), (8), and (9), and the transfer functions for the upper and lower sidebands result from replacing the factor for the carrier $\Phi=\omega_{0} L / c$ with $\Phi_{\text {low }}=$ $\left(\omega_{0}-\omega_{m}\right) L / c$ and $\Phi_{\text {up }}=\left(\omega_{0}+\omega_{m}\right) L / c$, respectively, where $L$ is the cavity length and $c$ the speed of light. The error signal can then be calculated according to [14]. Note that for a modulation frequency far outside the linewidth of the cavity, the sidebands are correspondingly suppressed at the transmission port $\mathrm{T}$ at the end mirror. By contrast, at the two correlated reflection ports $\mathrm{C} 1$ and $\mathrm{C} 3$, the phase-modulated side- bands are reflected and a PDH signal can be extracted as described above.

\section{Asymmetric PDH Error Signals}

For a grating manufactured with the so-called $\eta_{2_{\min }}$ configuration, where $\eta_{2}=\eta_{2_{\min }}=\left(1-\rho_{0}\right) / 2$ and $\eta_{0}=$ $\eta_{0_{\max }}=\left(1+\rho_{0}\right) / 2$, the DC signals of a gratingcoupled cavity that are detected at the forwardreflection and backreflection ports $\mathrm{C} 3$ and $\mathrm{C} 1$ behave exactly like signals that are detected at the reflection and transmission ports of an impedance-matched linear Fabry-Perot cavity. On resonance $\left(\Phi=0^{\circ}\right)$, the DC signal at port $\mathrm{C} 1$ is maximal, while it is minimal on antiresonance $\left(\Phi=90^{\circ}\right)$. This feature is the groundwork for the applicability of three-port gratings as the coupling component to arm cavities in future gravitational wave detectors.

In practice, small deviations from the theoretical minimal value $\eta_{2_{\min }}$ are almost unavoidable, since the boundaries derived in Eq. (5) are theoretical minimal/maximal values, and since fabricational inaccuracies still cannot be entirely excluded. However, numerous experimental and theoretical investigations have been carried out, and the theoretical description of the three-port grating has been validated [12,13,15]. Edgar et al. [17] have shown in a fully suspended environment at the Glasgow $10 \mathrm{~m}$ prototype interferometer that the $\mathrm{PDH}$ technique can be applied to a three-port grating cavity with an optimized dielectric three-port grating in $\eta_{2_{\min }}$ configuration. They could validate the remaining small asymmetry of the resonance shapes at the ports C1 and $\mathrm{C} 3$ and its influence on the symmetry of the PDH control signals. In that case the applicability of the control signal could be provided by applying a small offset to the demodulated error signal.

For larger deviations from the $\eta_{2_{\min }}$ configuration, the offset solution is no longer effective. As mentioned in Section 2, the output power at the two reflection ports $\mathrm{C} 1$ and $\mathrm{C} 3$ of a three-port grating cavity depends on the balance of $\eta_{0}$ and $\eta_{2}$, and in total the shapes of the resonance peaks are not symmetric to the detuning of the cavity. Similarly, the upper and lower sidebands obtain an asymmetric amplitude distribution with respect to the tuning. Hence, for an extraction at one of the two reflection ports $\mathrm{C} 1$ and $\mathrm{C} 3$, the strong asymmetry of the resonance profiles also occurs for the demodulated error signals. That results in a decreased slope of the error signal around the resonance frequency of the intracavity field and a consequentially decreased quality of the control circuit. However, since the power of the intracavity field and the incoming field combines partly constructively and partly destructively at the two reflection ports, and of course energy conservation must hold, the extracted signals are correlated with the opposite sign. Hence, the sum of the asymmetric error signals extracted at the two reflection ports reconstructs a proper point-symmetric error signal for the stabilization of the cavity. 
Table 1. Measured Values of the Three-Port Grating

\begin{tabular}{cc}
\hline Parameter & Measured Value \\
\hline$\eta_{0}^{2}$ & $78.2( \pm 4.0) \%$ \\
$\eta_{1}^{2}$ & $1.71( \pm 0.09) \%$ \\
$\eta_{2}^{2}$ & $19.47( \pm 0.95) \%$ \\
$\rho_{0}^{2}$ & $96.45( \pm 0.11) \%$ \\
Loss & $0.13( \pm 0.18) \%$ \\
\hline
\end{tabular}

\section{Experimental Procedure and Results}

To also validate the three-port grating description for strong asymmetries and the resulting asymmetric error signals, a customized dielectric three-port grating was manufactured at the Insitute of Applied Physics in Jena, Germany. The grating design was chosen such that the second-order diffraction efficiency is far away from the upper and lower boundaries [Eq. (5)]. The grating was manufactured with period $d=\overline{1} 450 \mathrm{~nm}$ for a first-order diffraction angle of $0^{\circ}$ and a second-order Littrow incident angle of $47.2^{\circ}$ at a laser wavelength of $1064 \mathrm{~nm}$ [18]. Initially the grating was characterized via a finesse measurement using the setup discussed in [12], leading to the diffraction efficiencies for $s$-polarized light given in Table 1.

A schematic of the experimental setup is shown in Fig. 1. The laser light at a wavelength of $1064 \mathrm{~nm}$ from a commercially available light source (Model Mephisto from InnoLight) was spatially filtered using a mode cleaner [19]. The cavity with the length of $L=44.8 \mathrm{~cm}$ was set up. The highly reflective cavity end mirror, with the amplitude transmittance $\tau^{2}=300 \pm 30 \mathrm{ppm}$ and the radius of curvature of $R_{c}=50.0 \mathrm{~cm}$, was mounted on a piezoelectric transducer (PZT) to linearly sweep the cavity length. For the generation of the PDH error signals, the light was phase modulated at a frequency of $f_{\text {mod }}=$ $15 \mathrm{MHz}$ by an electro-optical modulator (EOM). This frequency is well outside the measured linewidth of the cavity of FWHM $=1.94 \pm 0.06 \mathrm{MHz}$. At each port the signal was detected with a photo diode (PD1 and PD3), demodulated at the modulation frequency (M1 and M3), and low-pass filtered. The demodulated signals were electronically added to sum up to a symmetrical PDH signal. In Fig. 3 the DC signals at the ports are shown, including the normalized intracavity field detected in the transmission of the mirror when varying the cavity linearly with the PZT by more than one free spectral range. Clearly the asymmetry occurs at the ports $\mathrm{C} 1$ and $\mathrm{C} 3$, whereas the profile of the intracavity field shows the well known symmetric behavior. In order to adjust the signal strength at the forward-reflection and backreflection ports for the summation, a polarizing beam splitter and a $\lambda / 2$ plate was placed in front of each photodetector (see Fig. 1). By careful choice of the demodulation phase $\left(\phi=\phi_{0}\right)$, two signals could be generated that sum up to exactly reconstruct a symmetrical PDH signal.
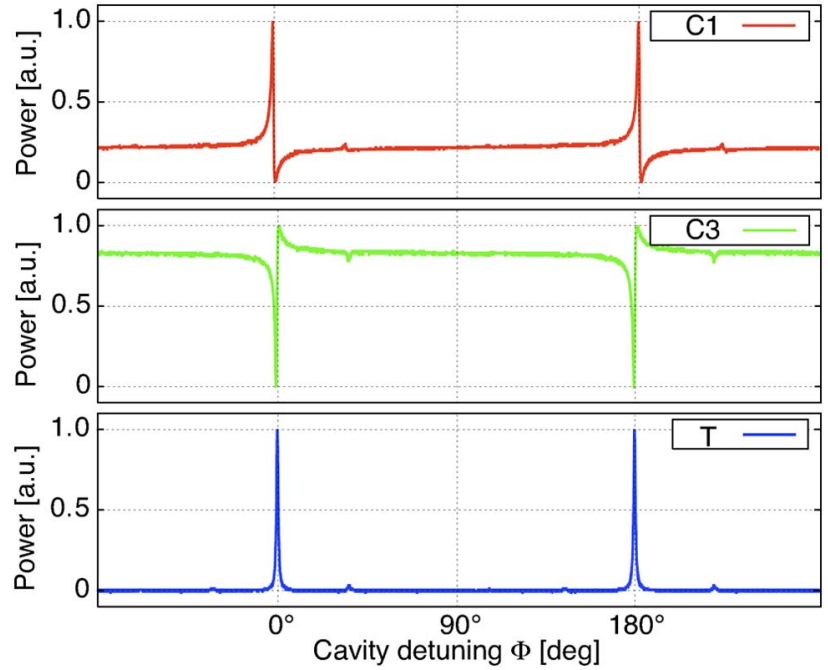

Fig. 3. (Color online) Normalized powers at the three output ports of the cavity $\mathrm{C} 1, \mathrm{C} 3$, and $\mathrm{T}$ with respect to the detuning $\Phi$ of the cavity. The resonance peaks at the reflected ports have an asymmetric shape that is not proportional to the intracavity power. The intracavity power is displayed by the transmitted intracavity field at port $\mathrm{T}$.

Figure $\underline{4}$ shows the generated signals at the ports C1 (red) and C3 (green) and the symmetrical sum (blue). Since both ports' signals were demodulated with the same local oscillator source, it was possible to adjust the two orthogonal phases, "in phase" $\left(\phi=\phi_{0}\right)$ and "quadrature phase" $\left(\phi=\phi_{0}+\pi / 2\right)$, at
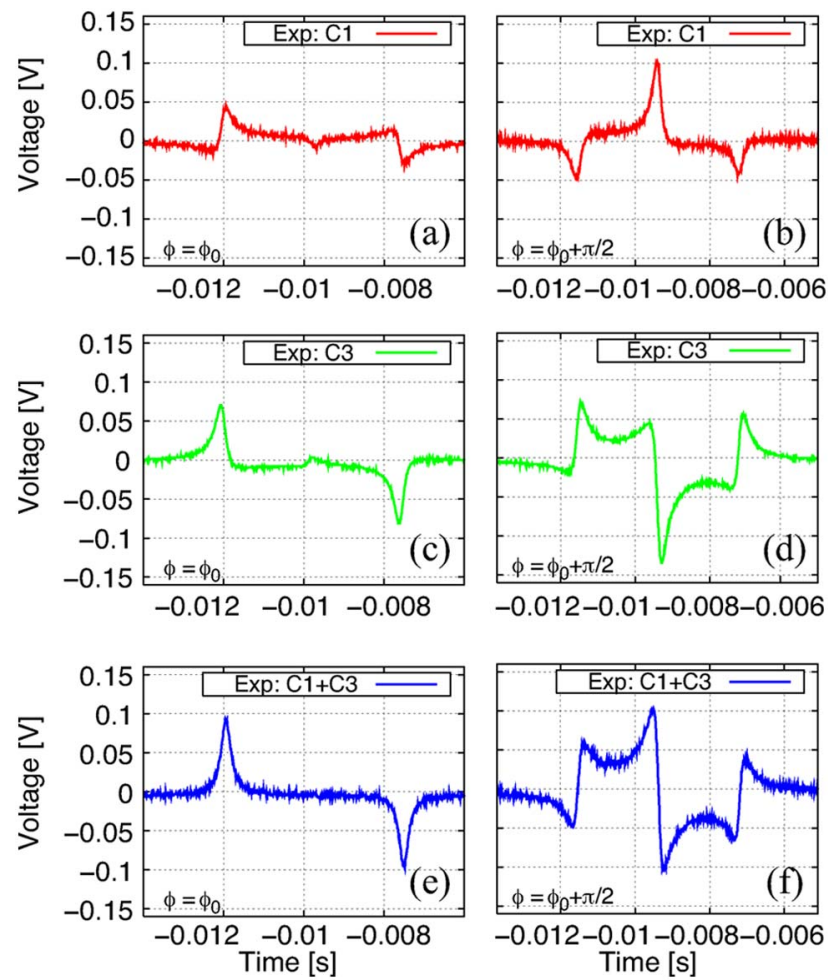

Fig. 4. (Color online) Measured error signals ("in-phase" left column, "quadrature-phase" right column) at (a), (b) the backreflection port and (c), (d) the forward-reflection port. (e), (f) The sum of the two signals provides a symmetrical PDH error signal. 

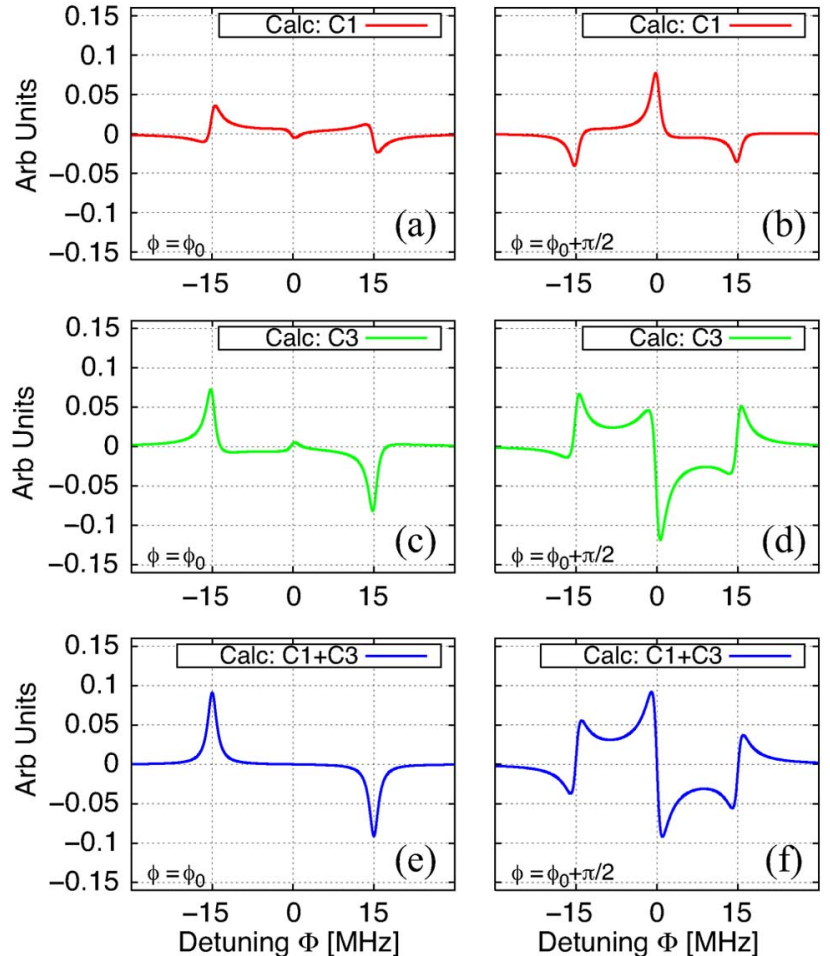

Fig. 5. (Color online) Calculated error signals ("in-phase" left column, "quadrature-phase" right column) at (a), (b) the backreflection port and (c), (d) the forward-reflection port and (e), (f) the sum of the two signals. The calculated signals are based on the measured grating values and show good agreement with the experimentally obtained error signals.

both ports simultaneously by varying the local oscillator phase as shown in the right column of Fig. 4. With the sum-signal of Fig. 4(f), a stable length control of the three-port grating cavity could be achieved. Note that the error signal derived from both cavity outputs has a slope that is steeper than possible error signals derived from just one cavity output port.

Based on the values of Table 1 , a calculation of the error signals was carried out. In Fig. 5 the calculated error signals are presented. In good qualitative and quantitative agreement, the calculated error signals confirmed the experimental results. Our simulations also showed that the presented method provides a symmetric, high-quality PDH error signal for cavity length control for any parameters of the three-port grating.

\section{Conclusion}

For all realistic three-port gratings, laser light that is reflected from a single port of a three-port grating coupled cavity is not proportional to the intracavity power. Accordingly, PDH error signals for cavity length control are not of optimum quality when derived from just one cavity port. This effect can only be avoided in the asymptotical limit of a three-port grating having an extremal value of its second-order diffraction efficiency $\eta_{2}$. We have demonstrated an extended PDH control scheme for three-port grating coupled cavities that copes with this problem for a realistic three-port grating. In our experiment we used a three-port grating that was designed and manufactured such that the value for its secondorder diffraction efficiency significantly deviated from its lower and upper theoretical boundaries. By extracting a signal at both reflection ports and electronic addition of the demodulated signals, a point-symmetric error signal could be generated that allows for stable length control of the cavity. We compared our results with simulated error signals and conclude that a proper PDH error signal can always be constructed, independent of the three-port grating's diffraction efficiencies.

This work has been supported by the Deutsche Forschungsgemeinschaft (DFG) within the Sonderforschungsbereich TR7, the Excellence Cluster QUEST, the IMPRS on Gravitational Wave Astronomy, and the ET design study supported by the European Commission under FP7 (Grant Agreement 211743)

\section{References}

1. B. P. Abbott, R. Abbott, R. Adhikari, P. Ajith, B. Allen, G. Allen, R. S. Amin, S. B. Anderson, W. G. Anderson, M. A. Arain, M. Araya, H. Armandula, P. Armor, Y. Aso, S. Aston, P. Aufmuth, C. Aulbert, S. Babak, P. Baker, S. Ballmer, C. Barker, D. Barker, B. Barr, P. Barriga, L. Barsotti, M. A. Barton, I. Bartos, R. Bassiri, M. Bastarrika, B. Behnke, M. Benacquista, J. Betzwieser, P. T. Beyersdorf, I. A. Bilenko, G. Billingsley, R. Biswas, E. Black, J. K. Blackburn, L. Blackburn, D. Blair, B. Bland, T. P. Bodiya, L. Bogue, R. Bork, V. Boschi, S. Bose, P. R. Brady, V. B. Braginsky, J. E. Brau, D. O. Bridges, M. Brinkmann, A. F. Brooks, D. A. Brown, A. Brummit, G. Brunet, A. Bullington, A. Buonanno, O. Burmeister, R. L. Byer, L. Cadonati, J. B. Camp, J. Cannizzo, K. C. Cannon, J. Cao, L. Cardenas, S. Caride, G. Castaldi, S. Caudill, M. Cavagli, C. Cepeda, T. Chalermsongsak, E. Chalkley, P. Charlton, S. Chatterji, S. Chelkowski, Y. Chen, N. Christensen, C. T. Y. Chung, D. Clark, J. Clark, J. H. Clayton, T. Cokelaer, C. N. Colacino, R. Conte, D. Cook, T. R. C. Corbitt, N. Cornish, D. Coward, D. C. Coyne, J. D. E. Creighton, T. D. Creighton, A. M. Cruise, R. M. Culter, A. Cumming, L. Cunningham, S. L. Danilishin, K. Danzmann, B. Daudert, G. Davies, E. J. Daw, D. DeBra, J. Degallaix, V. Dergachev, S. Desai, R. DeSalvo, S. Dhurandhar, M. Daz, A. Dietz, F. Donovan, K. L. Dooley, E. E. Doomes, R. W. P. Drever, J. Dueck, I. Duke, J.-C. Dumas, J. G. Dwyer, C. Echols, M. Edgar, A. Effler, P. Ehrens, E. Espinoza, T. Etzel, M. Evans, T. Evans, S. Fairhurst, Y. Faltas, Y. Fan, D. Fazi, H. Fehrmenn, L. S. Finn, K. Flasch, S. Foley, C. Forrest, N. Fotopoulos, A. Franzen, M. Frede, M. Frei, Z. Frei, A. Freise, R. Frey, T. Fricke, P. Fritschel, V. V. Frolov, M. Fyffe, V. Galdi, J. A. Garofoli, I. Gholami, J. A. Giaime, S. Giampanis, K. D. Giardina, K. Goda, E. Goetz, L. M. Goggin, G. Gonzlez, M. L. Gorodetsky, S. Goler, R. Gouaty, A. Grant, S. Gras, C. Gray, M. Gray, R. J. S. Greenhalgh, A. M. Gretarsson, F. Grimaldi, R. Grosso, H. Grote, S. Grunewald, M. Guenther, E. K. Gustafson, R. Gustafson, B. Hage, J. M. Hallam, D. Hammer, G. D. Hammond, C. Hanna, J. Hanson, J. Harms, G. M. Harry, I. W. Harry, E. D. Harstad, K. Haughian, K. Hayama, J. Heefner, I. S. Heng, A. Heptonstall, M. Hewitson, S. Hild, E. Hirose, D. Hoak, K. A. Hodge, K. Holt, D. J. Hosken, J. Hough, D. Hoyland, B. Hughey, S. H. Huttner, D. R. Ingram, T. Isogai, M. Ito, A. Ivanov, B. Johnson, W. W. Johnson, D. I. Jones, 
G. Jones, R. Jones, L. Ju, P. Kalmus, V. Kalogera, S. Kandhasamy, J. Kanner, D. Kasprzyk, E. Katsavounidis, K. Kawabe, S. Kawamura, F. Kawazoe, W. Kells, D. G. Keppel, A. Khalaidovski, F. Y. Khalili, R. Khan, E. Khazanov, P. King, J. S. Kissel, S. Klimenko, K. Kokeyama, V. Kondrashov, R. Kopparapu, S. Koranda, D. Kozak, B. Krishnan, R. Kumar, P. Kwee, P. K. Lam, M. Landry, B. Lantz, A. Lazzarini, H. Lei, M. Lei, N. Leindecker, I. Leonor, C. Li, H. Lin, P. E. Lindquist, T. B. Littenberg, N. A. Lockerbie, D. Lodhia, M. Longo, M. Lormand, P. Lu, M. Lubinski, A. Lucianetti, H. Lück, B. Machenschalk, M. MacInnis, M. Mageswaran, K. Mailand, I. Mandel, V. Mandic, S. Mrka, Z. Mrka, A. Markosyan, J. Markowitz, E. Maros, I. W. Martin, R. M. Martin, J. N. Marx, K. Mason, F. Matichard, L. Matone, R. A. Matzner, N. Mavalvala, R. McCarthy, D. E. McClelland, S. C. McGuire, M. McHugh, G. McIntyre, D. J. A. McKechan, K. McKenzie, M. Mehmet, A. Melatos, A. C. Melissinos, D. F. Menndez, G. Mendell, R. A. Mercer, S. Meshkov, C. Messenger, M. S. Meyer, J. Miller, J. Minelli, Y. Mino, V. P. Mitrofanov, G. Mitselmakher, R. Mittleman, O. Miyakawa, B. Moe, S. D. Mohanty, S. R. P. Mohapatra, G. Moreno, T. Morioka, K. Mors, K. Mossavi, C. MowLowry, G. Mueller, H. Müller-Ebhardt, D. Muhammad, S. Mukherjee, H. Mukhopadhyay, A. Mullavey, J. Munch, P. G. Murray, E. Myers, J. Myers, T. Nash, J. Nelson, G. Newton, A. Nishizawa, K. Numata, J. O'Dell, B. O'Reilly, R. O'Shaughnessy, E. Ochsner, G. H. Ogin, D. J. Ottaway, R. S. Ottens, H. Overmier, B. J. Owen, Y. Pan, C. Pankow, M. A. Papa, V. Parameshwaraiah, P. Patel, M. Pedraza, S. Penn, A. Perraca, V. Pierro, I. M. Pinto, M. Pitkin, H. J. Pletsch, M. V. Plissi, F. Postiglione, M. Principe, R. Prix, L. Prokhorov, O. Punken, V. Quetschke, F. J. Raab, D. S. Rabeling, H. Radkins, P. Raffai, Z. Raics, N. Rainer, M. Rakhmanov, V. Raymond, C. M. Reed, T. Reed, H. Rehbein, S. Reid, D. H. Reitze, R. Riesen, K. Riles, B. Rivera, P. Roberts, N. A. Robertson, C. Robinson, E. L. Robinson, S. Roddy, C. Röver, J. Rollins, J. D. Romano, J. H. Romie, S. Rowan, A. Rüdiger, P. Russell, K. Ryan, S. Sakata, L. Sancho de. la. Jordana, V. Sandberg, V. Sannibale, L. Santamara, S. Saraf, P. Sarin, B. S. Sathyaprakash, S. Sato, M. Satterthwaite, P. R. Saulson, R. Savage, P. Savov, M. Scanlan, R. Schilling, R. Schnabel, R. Schofield, B. Schulz, B. F. Schutz, P. Schwinberg, J. Scott, S. M. Scott, A. C. Searle, B. Sears, F. Seifert, D. Sellers, A. S. Sengupta, A. Sergeev, B. Shapiro, P. Shawhan, D. H. Shoemaker, A. Sibley, X. Siemens, D. Sigg, S. Sinha, A. M. Sintes, B. J. J. Slagmolen, J. Slutsky, J. R. Smith, M. R. Smith, N. D. Smith, K. Somiya, B. Sorazu, A. Stein, L. C. Stein, S. Steplewski, A. Stochino, R. Stone, K. A. Strain, S. Strigin, A. Stroeer, A. L. Stuver, T. Z. Summerscales, K.-X. Sun, M. Sung, P. J. Sutton, G. P. Szokoly, D. Talukder, L. Tang, D. B. Tanner, S. P. Tarabrin, J. R. Taylor, R. Taylor, J. Thacker, K. A. Thorne, A. Thüring, K. V. Tokmakov, C. Torres, C. Torrie, G. Traylor, M. Trias, D. Ugolini, J. Ulmen, K. Urbanek, H. Vahlbruch, M. Vallisneri, C. Van Den Broeck, M. V. van der Sluys, A. A. van Veggel, S. Vass, R. Vaulin, A. Vecchio, J. Veitch, P. Veitch, C. Veltkamp, A. Villar, C. Vorvick, S. P. Vyachanin, S. J. Waldman, L. Wallace, R. L. Ward, A. Weidner, M. Weinert, A. J. Weinstein, R. Weiss, L. Wen, S. Wen, K. Wette, J. T. Whelan, S. E. Whitcomb, B. F. Whiting, C. Wilkinson, P. A. Willems, H. R. Williams, L. Williams, B. Willke, I. Wilmut, L. Winkelmann, W. Winkler, C. C. Wipf, A. G. Wiseman, G. Woan, R. Wooley, J. Worden, W. Wu, I. Yakushin, H. Yamamoto, Z. Yan, S. Yoshida, M. Zanolin, J. Zhang, L. Zhang, C. Zhao, N. Zotov, M. E. Zucker, H. zur Mühlen, and J. Zweizig, "LIGO: the Laser Interferometer Gravitational-Wave Observatory," Rep. Prog. Phys. 72, 076901 (2009).

2. F. Acernese, P. Amico, M. Alshourbagy, F. Antonucci, S. Aoudia, S. Avino, D. Babusci, G. Ballardin, F. Barone, L.
Barsotti, M. Barsuglia, F. Beauville, S. Bigotta, S. Birindelli, M. A. Bizouard, C. Boccara, F. Bondu, L. Bosi, C. Bradaschia, S. Braccini, A. Brillet, V. Brisson, L. Brocco, D. Buskulic, E. Calloni, E. Campagna, F. Cavalier, R. Cavalieri, G. Cella, E. Cesarini, E. Chassande-Mottin, C. Corda, F. Cottone, A.-C. Clapson, F. Cleva, J.-P. Coulon, E. Cuoco, A. Dari, V. Dattilo, M. Davier, R. De Rosa, L. Di Fiore, A. Di Virgilio, B. Dujardin, A. Eleuteri, D. Enard, I. Ferrante, F. Fidecaro, I. Fiori, R. Flaminio, J.-D. Fournier, O. Francois, S. Frasca, F. Frasconi, A. Freise, L. Gammaitoni, F. Garufi, A. Gennai, A. Giazotto, G. Giordano, L. Giordano, R. Gouaty, D. Grosjean, G. Guidi, S. Hebri, H. Heitmann, P. Hello, L. Holloway, S. Karkar, S. Kreckelbergh, P. La Penna, M. Laval, N. Leroy, N. Letendre, M. Lorenzini, V. Loriette, M. Loupias, G. Losurdo, J.-M. Mackowski, E. Majorana, C. N. Man, M. Mantovani, F. Marchesoni, F. Marion, J. Marque, F. Martelli, A. Masserot, M. Mazzoni, L. Milano, C. Moins, J. Moreau, N. Morgado, B. Mours, A. Pai, C. Palomba, F. Paoletti, S. Pardi, A. Pasqualetti, R. Passaquieti, D. Passuello, B. Perniola, F. Piergiovanni, L. Pinard, R. Poggiani, M. Punturo, P. Puppo, K. Qipiani, P. Rapagnani, V. Reita, A. Remillieux, F. Ricci, I. Ricciardi, P. Ruggi, G. Russo, S. Solimeno, A. Spallicci, R. Stanga, R. Taddei, M. Tonelli, A. Toncelli, E. Tournefier, F. Travasso, G. Vajente, D. Verkindt, F. Vetrano, A. Vicere, J.-Y. Vinet, H. Vocca, M. Yvert, and Z. Zhang, "The Virgo status," Class. Quantum Grav. 23, S635-S642 (2006).

3. H. Grote (The LIGO Scientific Collaboration), "The status of GEO 600," Class. Quantum Grav. 25, 114043 (2008).

4. R. Takahashi (The TAMA Collaboration), "Status of TAMA," Class. Quantum Grav. 21, S403-S408 (2004).

5. M. Punturo, M. Abernathy, F. Acernese, B. Allen, N. Andersson, K. Arun, F. Barone, B. Barr, M. Barsuglia, M. Beker, N. Beveridge, S. Birindelli, S. Bose, L. Bosi, S. Braccini, C. Bradaschia, T. Bulik, E. Calloni, G. Cella, E. Chassande Mottin, S. Chelkowski, A. Chincarini, J. Clark, E. Coccia, C. Colacino, J. Colas, A. Cumming, L. Cunningham, E. Cuoco, S. Danilishin, K. Danzmann, G. De Luca, R. De Salvo, T. Dent, R. Derosa, L. Di Fiore, A. Di Virgilio, M. Doets, V. Fafone, P. Falferi, R. Flaminio, J. Franc, F. Frasconi, A. Freise, P. Fulda, J. Gair, G. Gemme, A. Gennai, A. Giazotto, K. Glampedakis, M. Granata, H. Grote, G. Guidi, G. Hammond, M. Hannam, J. Harms, D. Heinert, M. Hendry, I. Heng, E. Hennes, S. Hild, J. Hough, S. Husa, S. Huttner, G. Jones, F. Khalili, K. Kokeyama, K. Kokkotas, B. Krishnan, M. Lorenzini, H. Lück, E. Majorana, I. Mandel, V. Mandic, I. Martin, C. Michel, Y. Minenkov, N. Morgado, S. Mosca, B. Mours, H. Müller-Ebhardt, P. Murray, R. Nawrodt, J. Nelson, R. Oshaughnessy, C. D. Ott, C. Palomba, A. Paoli, G. Parguez, A. Pasqualetti, R. Passaquieti, D. Passuello, L. Pinard, R. Poggiani, P. Popolizio, M. Prato, P. Puppo, D. Rabeling, P. Rapagnani, J. Read, T. Regimbau, H. Rehbein, S. Reid, L. Rezzolla, F. Ricci, F. Richard, A. Rocchi, S. Rowan, A. Rüdiger, B. Sassolas, B. Sathyaprakash, R. Schnabel, C. Schwarz, P. Seidel, A. Sintes, K. Somiya, F. Speirits, K. Strain, S. Strigin, P. Sutton, S. Tarabrin, J. van den Brand, C. van Leewen, M. van Veggel, C. van den Broeck, A. Vecchio, J. Veitch, F. Vetrano, A. Vicere, S. Vyatchanin, B. Willke, G. Woan, P. Wolfango, and K. Yamamoto, "The third generation of gravitational wave observatories and their science reach," Class. Quantum Grav. 27, 084007 (2010).

6. K. A. Strain, K. Danzmann, J. Mizuno, P. G. Nelson, A. Rüdiger, R. Schilling, and W. Winkler, "Thermal lensing in recycling interferometric gravitational-wave detectors," Phys. Lett. A 194, 124-132 (1994).

7. V. B. Braginsky, M. L. Gorodetsky, and S. P. Vyatchanin, "Thermo-refractive noise in gravitational wave antennae," Phys. Lett. A 271, 303-307 (2000). 
8. K. Kuroda, M. Ohashi, S. Miyoki, D. Tatsumi, S. Sato, H Ishizuka, M.-K. Fujimoto, S. Kawamura, R. Takahashi, T. Yamazaki, K. Arai, M. Fukushima, K. Waseda, S. Telada, A. Ueda, T. Shintomi, A. Yamamoto, T. Suzuki, Y. Saito, T. Haruyama, N. Sato, K. Tsubono, K. Kawabe, M. Ando, K. Ueda, H. Yoneda, M. Musha, N. Mio, S. Moriwaki, A. Araya, N. Kanda, and M. E. Tobar, "Large-scale cryogenic gravitational wave telescope," Int. J. Mod. Phys. D 8, 557-579 (1999).

9. R. Nawrodt, A. Zimmer, T. Koettig, T. Clausnitzer, A. Bunkowski, E. B. Kley, R. Schnabel, K. Danzmann, S. Nietzsche, W. Vodel, A. Tünnermann, and P. Seidel, "Mechanical Q-factor measurements on a test mass with a structured surface," New J. Phys. 9, 225-234 (2007).

10. R. W. P. Drever, "Concepts for extending the ultimate sensitivity of interferometric gravitational wave detectors using non-transmissive optics with diffractive or holographic coupling," in Proceedings of the Seventh Marcel Grossman Meeting on General Relativity, R. T. Jantzen, G. M. Keiser, and R. Ruffini, eds., (World Scientific, 1995), pp. 1401-1406.

11. K.-X. Sun and R. L. Byer, "All-reflective Michelson, Sagnac, and Fabry-Perot interferometers based on grating beam splitters," Opt. Lett. 23, 567-569 (1998).

12. A. Bunkowski, O. Burmeister, P. Beyersdorf, K. Danzmann, R. Schnabel, T. Clausnitzer, E.-B. Kley, and A. Tünnermann, "Low-loss grating for coupling to a high-finesse cavity," Opt. Lett. 29, 2342-2344 (2004).
13. A. Bunkowski, O. Burmeister, K. Danzmann, and R. Schnabel, "Input-output relations for a 3-port grating coupled Fabry-Perot cavity," Opt. Lett. 30, 1183-1185 (2005).

14. E. D. Black, "An introduction to Pound-Drever-Hall laser frequency stabilization," Am. J. Phys. 69, 79-87 (2001).

15. A. Bunkowski, O. Burmeister, K. Danzmann, R. Schnabel, T. Clausnitzer, E.-B. Kley, and A. Tünnermann, "Demonstration of three-port grating phase relations," Opt. Lett. 31, 2384-2386 (2006).

16. O. Burmeister, M. Britzger, A. Thüring, D. Friedrich, F. Brückner, K. Danzmann, and R. Schnabel, "All-reflective coupling of two optical cavities with 3-port diffraction gratings," Opt. Express 18, 9119-9132 (2010).

17. M. P. Edgar, B. W. Barr, J. Nelson, M. V. Plissi, K. A. Strain, O. Burmeister, M. Britzger, K. Danzmann, R. Schnabel, T. Clausnitzer, F. Brückner, E.-B. Kley, and A. Tünnerman, "Experimental demonstration of a suspended diffractively coupled optical cavity," Opt. Lett. 34, 3184-3186 (2009).

18. T. Clausnitzer, E.-B. Kley, A. Tünnermann, A. Bunkowski, O. Burmeister, R. Schnabel, K. Danzmann, S. Gliech, and A. Duparré, "Ultra low-loss low-efficiency diffraction gratings," Opt. Express 13, 4370-4378 (2005).

19. B. Willke, N. Uehara, E. K. Gustafson, and R. L. Byer, "Spatial and temporal filtering of a $10 \mathrm{~W}$ Nd:YAG laser with a Fabry-Perot ring-cavity premode cleaner," Opt. Lett. 23, 1704-1706 (1998). 